\title{
Implantación del Método del Caso en la enseñanza del Periodismo en las universidades españolas: un análisis a partir de las guías docentes
}

\author{
Carmen FuenTE COBO \\ Centro Universitario Villanueva (adscrito a la Universidad Complutense de Madrid) \\ cfuentecobo@villanueva.edu \\ Montse MERA FernándeZ \\ Centro Universitario Villanueva (adscrito a la Universidad Complutense de Madrid) \\ mmera@villanueva.edu
}

Recibido: $15 / 10 / 2012$

Aceptado: 23/01/2013

\section{Resumen}

El Método del Caso es una herramienta pedagógica con escasa tradición en la enseñanza del Periodismo en España. Sin embargo, tras la implantación de las nuevas titulaciones de grado adaptadas al EEES, esta metodología de aprendizaje activo está suscitando un interés creciente en las Facultades de Comunicación / Información, donde se empieza a utilizar de una manera que no siempre responde al concepto, condiciones y objetivos de la propia metodología. Este artículo describe -a partir del análisis de las guías docentes de las asignaturas integradas en los planes de estudio- el grado de implantación del Método del Caso en las titulaciones de Periodismo impartidas en universidades españolas.

Palabras clave: Método del Caso, Enseñanza del Periodismo en España, Guías Docentes

\section{Implantation of Case Method in Journalism Studies in Spain. An Analysis Based on Teaching Guides}

\begin{abstract}
Case Method is mostly used in educational fields such as Management, Law, Medicine, Public Government or Education but it has less tradition in teaching Journalism. With the implementation of the new university degrees adapted to the European Space for Higher Education, Case Method and other active learning methodologies arouse an increasing interest within the Journalism academic community in Spain, where these techniques are being incorporated in a manner which not always respects the concept, conditions and objectives of traditional Case Method. This article describes the level of implementation of Case Method in Spain's faculties of Journalism, based on the analysis of the teaching guides of subjects included in the teaching programs of these faculties.
\end{abstract}

Keywords: Case Method, Journalism studies in Spain, teaching guides

\section{Referencia normalizada}

FUENTE COBO, Carmen y MERA FERNÁNDEZ, Montse (2013): "Implantación del Método del Caso en la enseñanza del Periodismo en las universidades españolas: un análisis a partir de las guías docentes". Estudios sobre el Mensaje Periodístico. Vol. 19, Núm. especial marzo, págs.: 177-186. Madrid, Servicio de Publicaciones de la Universidad Complutense.

Sumario: 1. Introducción. 2. Metodología. 3. Desarrollo. 4. Conclusiones 5. Referencias bibliográficas.

\section{Introducción}

Utilizado inicialmente en la enseñanza de la Medicina y después en la del Derecho, el Método del Caso saltó a principios del siglo XX a los programas de formación en negocios y gestión empresarial de la Harvard Business School, que utiliza este método 
en más del 90\% de sus clases y que lo entiende como parte de una aproximación global a la educación y desarrollo de la gestión empresarial, lo que implica a su vez la necesidad de plantear su uso de manera bien orquestada con otras aproximaciones metodológicas. En este sentido, el método se construye en torno a los conceptos de "metáfora" y "simulación" y parte de la idea de que la gestión empresarial es más una destreza para descifrar problemas y plantear soluciones que una colección de técnicas o conocimientos. Cada caso es una metáfora de un tipo de problemas determinados. Y puesto que la mejor manera de adquirir una destreza es practicar en procesos de simulación, para decidir correctamente es preciso ejercitar muchas veces dicha capacidad y los procesos implicados ${ }^{1}$.

Si el empleo de casos como método pedagógico requiere su inserción en modelos de organización docente más amplios, en los que todos los recursos y metodologías deben alinearse en función de los objetivos docentes perseguidos, en el caso de la formación universitaria de los periodistas nos encontramos con un problema inicial de alto calado: la falta de consenso en torno a cuál debe ser el contenido de dicha formación y, por ende, qué aproximaciones pedagógicas son más adecuadas. Esta ausencia de consenso es global, como ponen de manifiesto estudios sobre educación comparada (MacDonald, 2006; Mensing, 2010).

De la misma manera, es igualmente general el acuerdo en la comunidad académica internacional sobre la necesidad de dedicar una atención prioritaria a la formación de los periodistas, no sólo como condición y soporte de su propia identidad sino, sobre todo y por encima de todo, por la función que desempeña la información (como facilitadora del sentido de integración y pertenencia a la comunidad) para todo ciudadano, en la visión optimista y generosa de Rosenstiel y Kovach sobre los públicos interconectados (2003: 38-46), y de generación de sentido sobre la realidad que nos rodea (Casals, 2006: 59-70). Esto es lo que significa el "periodismo esencial" cuyo fin último -recordaba Enrique de Aguinaga- no es la información sino "el conocimiento por depuración de la información" (2002: 169). Este periodista esencial, "que lo será en cualquier circunstancia y en cualquier tiempo, es el periodista que la Universidad tiene la responsabilidad de perfilar. Este debería ser el periodista del Siglo XXI" (Aguinaga, 2002: 170).

¿Qué tipo de formación necesita, entonces, el periodista de las generaciones actuales y, más aún, el de las venideras?, ¿cómo hay que formar al profesional de lo que Rosenstiel y Kovach denominan Next Journalism (2010)? Aunque es cierto que por más que se transforme el contexto tecnológico, económico y social en el que se produce y distribuye la información, la esencia del periodismo y sus funciones permanece inalterado, también hay que tener en cuenta que, evidentemente, el nuevo escenario demanda nuevas habilidades y competencias de los profesionales dedicados a lo que Aguinaga denomina periodismo esencial o al periodismo de verificación, en equivalencia no plena pero sí próxima, de Rosenstiel y Kovach.

1 Harvard Business School. An introduction to Cases. 9-584-097. Rev. October 12,1988. [www.ecch.com, consultado el 10 de junio de 2012] 
En su declaración sobre el futuro de la formación de los periodistas, realizada tras concluir los trabajos de la comisión puesta en marcha para analizar cómo debería adaptarse la prestigiosa Escuela de Periodismo de la Universidad de Columbia en Nueva York, el rector de esta universidad, Lee Bollinger (2003), señalaba que una escuela profesional de Periodismo -no olvidemos que la Columbia School of Journalism sólo imparte titulaciones de posgrado- enfocada a las necesidades de los tiempos presentes y futuros debe ser capaz de ayudar a sus alumnos, entre otras cosas, a adquirir las habilidades y hábitos intelectuales necesarios para enfrentarse a situaciones nuevas y cambiantes, aprendiendo a "pensar como periodistas" en todo tipo de contextos y situaciones.

Este tipo de hábitos intelectuales requieren, sin duda, enfoques docentes innovadores y una mejor atención a la formación en la toma de decisiones, objetivo para el que la metodología basada en casos resulta especialmente adecuada. Marcial Murciano, presidente honorario de la Conferencia de Decanos de Comunicación / Información, expresa así este reto: "No bastará con una sólida formación teórica, científica y práctica, sino que será necesario ampliar constantemente este conocimiento con el análisis continuo de casos prácticos". (Murciano, 2006: 89-100)

\section{Metodología}

Para analizar el nivel de implantación del Método del Caso en la enseñanza del Periodismo en España, en esta primera fase de descripción del estado de la cuestión nos hemos centrado exclusivamente en la información publicada sobre la metodología docente seguida en cada asignatura y materia contenidas en los planes de estudio de todas las titulaciones de Grado en Periodismo impartidas en universidades españolas. En este sentido es preciso recordar que la información sobre la metodología docente es uno de los contenidos que deben hacer públicas todas las universidades en relación con los títulos de Grado implantados, en cumplimiento de las exigencias que en materia de transparencia se derivan del EEES.

De este modo, se han identificado 41 centros universitarios en los que, de acuerdo con la información contenida en la Guía Oficial de Titulaciones y Postgrados de las Universidades Españolas publicado por la CRUE, se han impartido títulos de Grado en Periodismo en el curso 2011-2012. Para cada una de dichas universidades se estudiaron los programas y las guías docentes de las titulaciones identificadas, lo que supuso la revisión de 150 programas y guías docentes. ${ }^{2}$

Para proceder a la observación del nivel de implantación del Método del Caso, en esta primera fase se extrajeron de los programas y guías docentes todas la referencias

2 Se ha procedido a una segunda revisión de las guías docentes publicadas por las distintas universidades, ya que la primera revisión -de cuyos resultados se daba cuenta en la comunicación presentada al congreso CUICIID 2012 y que había sido efectuada en los dos meses finales del primer semestre de 2012- muchas universidades no habían actualizado esta información o no permitían el acceso a la misma ( $20 \%$ de accesos fallidos). La lectura que presentamos corresponde a la información completada durante los meses de septiembre y octubre de 2012. 
a casos contenidas en los mismos, tanto si el concepto "Método del Caso" aparecía explícito en el apartado de metodologías docentes como si simplemente se enunciaba de manera genérica la utilización de casos como parte del proceso pedagógico.

Una vez obtenido el listado de asignaturas, se procedió a agruparlas por materias, de acuerdo con la estructura general del Título de Grado en Periodismo contenida en el Libro Blanco de ANECA en relación con los contenidos comunes obligatorios $(50 \%$ del total).

\section{Desarrollo}

La mayor parte de los autores coinciden en señalar que la denominación de Método del Caso se utiliza para referirse a un conjunto variado de técnicas y herramientas docentes utilizadas en el aula y que se caracterizan por estar basadas en la descripción de situaciones tomadas de la vida real.

Las escuelas de negocio con mayor veteranía y tradición en el uso de esta metodología coinciden, sin embargo, en una aproximación al concepto que atiende principalmente a la implicación del alumno en el proceso como elemento definitorio del método empleado. James G. Clanson, de la Universidad de Darden, en Virginia (que junto con la Harvard Business School y la Western Ontario University de Canadá constituye el triunvirato de las universidades norteamericanas de prestigio caracterizadas por el uso intensivo de este método), recuerda que en el "método clásico" el profesor o instructor no habla más del 15\% del tiempo que está con sus alumnos. En el otro extremo se sitúan los profesores que denominan "Método del Caso" al uso de materiales escritos o audiovisuales como elementos de ilustración o de análisis en sus clases, mientras mantienen el tono y el discurso de la sesión y continúan siendo la voz dominante. ${ }^{3}$

Para Clanson, las clases en las que el profesor habla más del 50\% del tiempo, aunque utilice casos para ilustrar o como base para el análisis, deberían llamarse clases de discusión o clases magistrales (lectures). La esencia del método reside, por tanto, en que debe estar construido como descripción de situaciones y problemas de la vida profesional en torno a los cuales el alumno debe posicionarse (orientación fundamental a la decisión), y que exija un alto nivel de participación e implicación personal de los alumnos.

E. Raymond Corey, de la Harvard Business School, coincide con este planteamiento aunque no de manera tan radical, y señala que las diferencias que pueden encontrarse en el mundo universitario a la hora de aproximarse al método se refieren, fundamentalmente, a las siguientes cuestiones:

- Grado de input de los alumnos vs. profesor

- Modelo de relación profesor-estudiante y estudiante-estudiante

- Hasta qué punto hay dirección del instructor vs. agenda-setting por parte de los propios alumnos

3 CLANSON, James G.: “Case Method. Darden Business Publishing”. University of Virginia. UVA-PHA-0032. [www.eech.com, consultado el 10 de junio de 2012) 
- Hasta qué punto el profesor cierra el caso en forma de lección final, vs. dejar que sean los propios alumnos los que describan y sinteticen las lecciones aprendidas (Corey, 1998).

En general, por tanto, hay cierta coincidencia en que lo que diferencia el Método del Caso de otras metodologías activas como el Aprendizaje Cooperativo y el Aprendizaje Basado en Problemas es la capacidad del profesor para conseguir que el alumno se posicione respecto a un hecho concreto (Benito y Cruz, 2007, 54-60). Desde el punto de vista formal, los pasos o fases habituales en el análisis de casos suelen ser las siguientes:

- Análisis y preparación individual

- Discusión en grupo informal

- Discusión en clase

- Finalización y generalización sobre lo que se ha aprendido

Puede observarse que el Método del Caso es aplicable a prácticamente todas las disciplinas, en la medida en que el objetivo de toda enseñanza no es tanto impartir conocimiento cuanto motivar a los alumnos para que se planteen las preguntas importantes en relación con una materia determinada, y que desarrollen los hábitos intelectuales que les permitan enfrentarse a problemas y encontrar soluciones (Schmenner, 2002).

Resulta evidente que la utilización del Método del Caso exige unas habilidades específicas por parte del profesor, así como una dedicación en términos de tiempo de preparación en muchas ocasiones superior a la de otras herramientas pedagógicas (Clanson calcula que preparar un caso para una sesión de 90 minutos supone de 8 a 15 horas).

Otra cuestión que conviene no perder de vista es el nivel de complejidad, pues no es lo mismo desarrollar un caso para estudiantes de grado que para alumnos de posgrado, como tampoco es lo mismo un estudiante de primer curso que uno de último curso.

Para algunos expertos, el criterio clave para decidir sobre el contenido y el tipo de caso es la motivación del alumno. Así, Scott Andrew, instructor del Método del Caso en diferentes universidades británicas, indica que al elegir casos para estudiantes de posgrado se inclina por aquellos que se centran en decisiones de gestión, mientras que para los estudiantes de grado procura que el tema sea atractivo, próximo y comprensible (Andrew, 2000).

El Método del Caso tiene una amplia tradición, sobre todo en el ámbito anglosajón, como herramienta pedagógica en la formación de los periodistas para enfrentarse a la resolución de situaciones dilemáticas relacionadas con la ética profesional. No solamente es abundante la literatura generada, sino que también se han desarrollado recursos docentes compartidos, de alta calidad y especialización, entre los que cabe destacar la base de casos del Poynter Institute, que facilita el acceso a análisis realizados por expertos -desde la perspectiva de la ética periodística- de situaciones reales. Pero, sobre todo, debe citarse el proyecto de la Columbia School of Journalism, 
el Case Consortium (anteriormente conocido como Knight Case Studies Initiative), que ha permitido generar un banco de casos para su uso en programas académicos de formación de periodistas y que está a disposición de la comunidad universitaria internacional. Un simple vistazo a esos casos permite apreciar que el nivel y complejidad de los mismos responde al propio carácter de la docencia, exclusivamente de posgrado, impartida en la Columbia University.

El abanico de disciplinas y materias cubiertas por los casos acumulados en la base del Case Consortium de la Universidad de Columbia permite advertir, por otra parte, hasta qué punto el recurso el Método del Caso ha evolucionado desde una concepción vinculada casi exclusivamente a la ética periodística hacia su uso en áreas de conocimiento relacionadas con la gestión y la toma de decisiones en el ámbito de la empresa periodística (gestión empresarial) y de los procesos de producción informativa (gestión de las redacciones).

En la bibliografía más reciente puede observarse la misma evolución. El Método del Caso se emplea en la actualidad para ilustrar y desarrollar la formación de la ética personal (Bugeja, 2008; Boeyink y Borden, 2010), como herramienta que permite enfocar el aprendizaje crítico en torno a principios centrales de la ética periodística (en este sentido, son especialmente interesantes los trabajos del profesor de la Universidad de Illinois Clifford G. Christians), como fundamento para la formación en competencias relacionadas con las nuevas tecnologías (Fried y Singer, 2007) y en la orientación de las decisiones que tienen que ver con la transformación de los medios en la actualidad, ámbito en el que destacan los trabajos publicados por la Universidad de Columbia (Rosenstiel y Mitchell, 2003; Lundberg y Palmer, 2011).

En España la literatura científica y académica dedicada a la descripción y planteamiento de casos periodísticos es todavía escasa, aunque gracias a la iniciativa de fundaciones como la valenciana Coso (impulsora de un Congreso Internacional sobre Ética y Derecho de la Información que se viene celebrando anualmente desde hace una década) podemos disponer de casos de interés contenidos en algunos de los volúmenes publicados por esta organización, a partir de las comunicaciones y ponencias presentadas cada año. También puede citarse en esta dirección la Conferencia Internacional sobre Ética de los Medios, organizada por la Universidad de Sevilla, y de la que en abril de 2013 se celebrará la segunda edición. Sin embargo, la revisión de las comunicaciones presentadas en la primera edición de este congreso permite comprobar en qué medida todavía es muy limitado el interés de investigadores y docentes por esta metodología. De las 105 comunicaciones presentadas y publicadas en actas en la edición de 2011 solamente una aludía al Método del Caso como metodología específica, si bien en muchas otras se utilizaba el concepto "caso" en su sentido más general ${ }^{4}$. Uno de los impulsores de este congreso, el profesor Juan Carlos Suárez Villegas, es autor de una monografía que aborda el análisis de casos aunque desde

${ }^{4}$ La ética de la comunicación a principios del siglo XXI. Libro de Actas del I Congreso Internacional de Ética de la Comunicación. Universidad de Sevilla, Facultad de Comunicación, 11-13 de marzo de 2011 [http://monitorando.files.wordpress.com/2011/04/libro-actas -congreso-etica-comunicación.pdf] 
una perspectiva que difícilmente podría denominarse, en sentido estricto, como propia del Método del Caso, ya que el autor construye más bien un modelo de análisis de noticias en función de criterios éticos (Suárez Villegas, 2011).

En al menos 31 de las 36 universidades a cuyas guías docentes y programas ha sido posible acceder, aparecen alusiones al uso de casos, bien como contenido docente o bien como metodología docente $(86 \%)$. Por otra parte, el recurso a esta herramienta no se ciñe a las materias tradicionales de Ética Periodística, sino que puede encontrarse relacionado con materias muy diversas, principalmente aquellas vinculadas con la formación en gestión de empresas y procesos de producción periodística.

Relación de asignaturas y materias en las que se utilizan casos

(contenidos comunes obligatorios)

\begin{tabular}{|c|c|}
\hline Materia (ANECA, Libro Blanco) & Asignatura \\
\hline $\begin{array}{l}\text { Expresión Oral y Escrita para los medios de in- } \\
\text { formación }\end{array}$ & Pragmática y Discurso en el Periodismo \\
\hline Información periodística y comunicación digital & $\begin{array}{l}\text { Redacción Periodística } \\
\text { Información y Comunicación Política } \\
\text { Información Periodística Especializada }\end{array}$ \\
\hline Organización y producción informativa & $\begin{array}{l}\text { Teoría de la Empresa Informativa } \\
\text { Documentación } \\
\text { Marketing aplicado al Periodismo } \\
\text { Gabinetes de Comunicación } \\
\text { Dirección y Gestión de Empresa Periodísticas }\end{array}$ \\
\hline Periodismo especializado & Literatura y Medios de Comunicación \\
\hline Teoría e Historia del Periodismo & Historia del Periodismo \\
\hline $\begin{array}{l}\text { Libertad de expresión, responsabilidad periodís- } \\
\text { tica, opinión pública }\end{array}$ & $\begin{array}{l}\text { Etica y Deontología Periodística } \\
\text { Derecho de la Información } \\
\text { Opinión Pública }\end{array}$ \\
\hline $\begin{array}{l}\text { Fundamentos y análisis de la información y la } \\
\text { comunicación }\end{array}$ & $\begin{array}{l}\text { Teoría y Práctica del Periodismo } \\
\text { Estructura y Sistema Mundial de la Información }\end{array}$ \\
\hline $\begin{array}{l}\text { Mundo actual: comprensión y evolución con- } \\
\text { temporánea }\end{array}$ & $\begin{array}{l}\text { Sociología } \\
\text { Relaciones Exteriores de España } \\
\text { Relaciones Internacionales }\end{array}$ \\
\hline
\end{tabular}

Por otra parte, del total de referencias que contienen la palabra "caso" obtenidas en el análisis, son muy escasas aquellas en las que aparece explicitado el "Método del Caso" como herramienta pedagógica, mientras que en el resto se recogen de manera general, en enunciados del tipo "uso de casos", como un elemento más del material didáctico empleado en el aula o fuera de ella. La terminología empleada incluye las siguientes denominaciones: "casos prácticos", "casos prácticos derivados de contenidos teóricos", "debate de casos", "descripción de casos", "casos teórico-prácticos", "análisis de casos", "resolución de casos", "estudios de caso", "metodología del estudio de casos" o "análisis de casos paradigmáticos".

Una cuestión sobre la que merece la pena detenerse es el propio diseño de las guías docentes estudiadas. Según se ha podido comprobar, en no pocos casos éstas incluyen apartados de metodología docente con opciones cerradas para los profesores, que deben subrayar aquellas metodologías que, de una u otra manera, entienden que van 
a utilizar durante el curso. Este tipo de entornos de descripción cerrada puede ser la causa de que en algunas universidades el elenco de asignaturas en las que figura la utilización del Método del Caso como metodología docente sea amplísimo y de que, tal vez, esto no responda de manera exacta a la realidad de la docencia impartida (así parece ponerlo de manifiesto la discrepancia entre metodologías elegidas y dedicación en horas que se señala en los cronogramas de desarrollo de las asignaturas).

\section{Conclusiones}

Del estudio realizado se deduce un interés claro y extendido por la comunidad docente de la titulación de Periodismo por la utilización de casos como herramienta pedagógica adaptada a las necesidades y objetivos de las asignaturas y materias propias de esta titulación.

Pero, de acuerdo con los datos obtenidos en esta primera exploración, los profesores de Periodismo utilizan el término "caso" para referirse a realidades muy diversas entre las que se incluye, de manera preferente, el recurso a descripciones de situaciones reales, como elemento ilustrativo de contenidos del temario.

\section{Referencias bibliográficas}

AGENCIA NACIONAL DE EVALUACIÓN DE LA CALIDAD Y ACREDITACIÓN (2005). Libro Blanco. Títulos de Grado en Comunicación. En: [http://www.aneca .es/var/media/150336/libroblanco_comunicacion_def.pdf.

AGUINAGA, Enrique (2002): "El periodista en el umbral del siglo XXI". Estudios sobre el Mensaje Periodístico, vol. 8, pp. 157-170. Madrid, Servicio de Publicaciones de la Universidad Complutense.

ANDREW, Scott: "Developing cases and clases for undergraduate teaching". ECCHO 24, Summer 2000. www.ecch.com/ECCHObackissues

BENITO, Águeda y CRUZ, Ana (2007): Nuevas claves para la docencia universitaria en el Espacio Europeo de Educación Superior. Narcea, S.A. de Ediciones, ( $2^{\mathrm{a}}$ ed).

BOEYINK, David E. \& BORDEN, Sandra L. (2010): Making Hard Choices in Journalism Ethics. Cases and Practices. New York \& London, Routledge

BOLLINGER, Lee C. (2003): "Statement on the Future of Journalism Education". April 15, en http://www.columbia.edu/cu/president/docs/communications/20022003/030415-journalism.html [fecha de consulta: 25 de octubre de 2012]

BUGEJA, Michael (2008): Living Ethics Across Media Platforms: New York, Oxford. Oxford University Press

CASALS CARRO, María Jesús (2006): "La enseñanza del periodismo y las nuevas tecnologías de la información y de la comunicación". Estudios sobre el Mensaje Periodístico, vol. 12, pp. 59-70. Madrid, Servicio de Publicaciones de la Universidad Complutense.

CHRISTIANS, Clifford G.; FACKLER, Mark; BRITTAIN, Kathy et alii (2009.): Media Ethics. Cases and Moral Reasoning. Pearson. 
CLANSON, James G.: "Case Method". Darden Business Publishing. University of Virginia. UVA-PHA-0032. [www.eech.com]

COREY, E Raymond: "Case Method Teaching". Harvard Business School. 9-581058. Rev Nov 6, 1998. www.eech.com

DE MIGUEL DÍAZ, Mario (Coord., 2006): Metodologías de enseñanza y aprendizaje para el desarrollo de competencias. Orientaciones para el profesorado universitario ante el Espacio Europeo de Educación Superior. Madrid, Alianza Editorial.

FRIEND, Cecilia; SINGER, James B. (2007): Online Journalism Ethics. Traditions and Transitions. New York, London, M.E. Sharpe

HARVARD BUSINESS SCHOOL (1988): “An introduction to Cases". 9-584-097. Rev. October 12,1988. [www.ecch.com]

KOVACH, Bill; ROSENSTIEL, Tom (2003): Los elementos del periodismo. Madrid, Ediciones Santillana, S.A.

KOVACH, Bill; ROSENSTIEL, Tom (2010): Blur. How to know what is true in the age of information overload. New York, Bloomsbury.

LUNDBERG, Kirsten; PALMER, Ruth (2011): Our digital future: Boardrooms and Newsrooms. Knight Case Studies Initiative. Graduate School of Journalism. Columbia University

MACDONALD, Isabel (2006): "Teaching Journalists to save the profession. A critical assessment of recent debates on the future of US and Canadian journalists education". Journalism Studies, 7, Issue 5, pp. 745-764 (Published online: 17 Feb 2007).

MENSING, Donica (2010): "Rethinking [again] the future of Journalism Education". Journalism Studies, vol. 11, Issue 4, pp. 511-523 (Published online: 08 Jul 2010)

PEARSON, Mark (1994): "Rethinking quality in Journalism Education". Australian Journalism Review, vol 16, $\mathrm{n}^{\mathrm{o}}$ 2, jun-dec., pp 67-72, en http://epublications.bond.edu.au/hss_pubs/81.

SCHMENNER, Roger W. (2003): “Thoughts on Case Teaching”. International Institute for Management Development. Lausanne. Switzerland. 2002. IMD-3-1052 (GM 1052) v.28.10.2003 (www.eech.com, recuperado 10.06.2012)

SUÁREZ VILLEGAS, Juan Carlos (2011): Análisis ético de la información. Sevilla, Editorial MAD.

VV.AA. (2011): La ética de la comunicación a principios del siglo XXI. Libro de Actas del I Congreso Internacional de Ética de la Comunicación. Sevilla, Universidad de Sevilla, Facultad de Comunicación, 11-13 de marzo de 2011. [http://monitorando.files.wordpress.com/2011/04] 


\section{Dra. Carmen FUENTE COBO}

Centro Universitario Villanueva (Universidad Complutense de Madrid) cfuentecobo@villanueva.edu Profesora de Periodismo

\section{Dra. Montse MERA FERNÁNDEZ}

Centro Universitario Villanueva (Universidad Complutense de Madrid) mmera@villanueva.edu

Profesora de Periodismo 